Cahiers $d u$ MONDE RUSSE

\section{Cahiers du monde russe}

Russie - Empire russe - Union soviétique et États indépendants

45/3-4 | 2004

Varia

\title{
Le symbolisme russe en quête de paradis originel
}

\section{Georges Nivat}

\section{OpenEdition \\ Journals}

Édition électronique

URL : https://journals.openedition.org/monderusse/8703

DOI : $10.4000 /$ monderusse. 8703

ISSN : $1777-5388$

Éditeur

Éditions de l'EHESS

Édition imprimée

Date de publication : 1 juillet 2004

Pagination : $569-578$

ISBN : 2-7132-2009-2

ISSN : $1252-6576$

\section{Référence électronique}

Georges Nivat, "Le symbolisme russe en quête de paradis originel », Cahiers du monde russe [En ligne], 45/3-4 | 2004, mis en ligne le 01 janvier 2007, consulté le 02 septembre 2022. URL : http:// journals.openedition.org/monderusse/8703 ; DOl : https://doi.org/10.4000/monderusse.8703 
chercher : repérer : avancer

Cet article est disponible en ligne à l'adresse :

http://www.cairn.info/article.php?ID REVUE=CMR\&ID NUMPUBLIE=CMR 453\&ID ARTICLE=CMR $453 \quad 0569$

\title{
Le symbolisme russe en quête de paradis originel
}

\author{
par GEORGES NIVAT
}

\section{Editions de l'EHESS | Cahiers du monde russe}

\author{
2004/3-4 - Vol 45 \\ ISSN 1252-6576 | ISBN 2713220092 | pages 569 à 578
}

Pour citer cet article :

—NIVAT G., Le symbolisme russe en quête de paradis originel, Cahiers du monde russe 2004/3-4, Vol 45, p. 569-578.

Distribution électronique Cairn pour les Editions de l'EHESS.

(C) Editions de l'EHESS. Tous droits réservés pour tous pays.

La reproduction ou représentation de cet article, notamment par photocopie, n'est autorisée que dans les limites des conditions générales d'utilisation du site ou, le cas échéant, des conditions générales de la licence souscrite par votre établissement. Toute autre reproduction ou représentation, en tout ou partie, sous quelque forme et de quelque manière que ce soit, est interdite sauf accord préalable et écrit de l'éditeur, en dehors des cas prévus par la législation en vigueur en France. Il est précisé que son stockage dans une base de données est également interdit. 


\section{LE SYMBOLISME RUSSE EN QUÊTE DE PARADIS ORIGINEL}

Dépasser la frontière entre les arts n'est pas propre au symbolisme russe, il a hérité cette ambition du romantisme allemand, et même on peut dire que l'ambition d'échapper au « fini » de l'art est aussi ancienne que l'art lui-même. Le non finito est propre à tous les grands arts in statu nascendi, il est à certains moments l'acmé que recherche l'artiste, même si son client cherche, lui, le finito : alors l'ébauche sera pour l'artiste et l'œuvre finie pour le client de l'artiste. Il y a pour Baudelaire le bon et le mauvais « vague ». Le bon vague permet de gommer les frontières artificielles, d'établir les correspondances entre le peintre et le poète, Delacroix et Baudelaire, entre l'architecture et la musique. On trouve chez Baudelaire non seulement le temple de la nature et la forêt de symboles du fameux sonnet tant commenté des « Correspondances », mais aussi la porosité entre la matière dure et l'effluve :

Il est de forts parfums pour qui toute matière

Est poreuse. On dirait qu'ils pénètrent le verre.

(Le flacon)

Troisième élément de cette mutation constante et insidieuse des choses : la Réversibilité, cette réversibilité qui est d'abord un concept catholique de la réversibilité des fautes depuis le premier Adam, et qui devient la fluctuation des âmes dans une grande économie du salut qu'il ne peut embrasser. Les «phares » de Baudelaire sont les grands artistes réversibles, Dante, Goya, Delacroix. Ceux pour qui l'échelle de l'Être peut se renverser, le beau embrasser le laid, le laid inclure le beau. Andrej Belyj, comme tous les autres symbolistes russes a été «baudelairien », mais la comparaison qu'il fait de Baudelaire avec Nietzsche est assez étonnante : Baudelaire représente la race romane, Nietzsche la race allemande (la génération symbo-

* Les trois textes réunis dans ce dossier ont fait l'objet d'exposés lors du colloque consacré au « Symbolisme en Russie » qui s'est tenu au musée des Beaux-Arts de Bordeaux en mai 2000. 
liste est nourrie de concepts de races) ; l'un, le Français, établit le dualisme de la contemplation, l'autre, l'Allemand, établit le primat de l'acte créateur. Et, en effet, l'influence de Nietzsche vient apporter une autre porosité entre les paramètres essentiels de la vie : la contamination entre art et vie. C'est d'ailleurs ce qui a fait que la génération symboliste russe a pu tant aduler le maître de Jasnaja Poljana, qui n'était pas tendre envers le symbolisme, comme on sait. Ce sont la fuite de Tolstoj hors du monde, sa théorie de l'art comme une contagion, enfin sa mort à Astapovo qui ont établi de façon prophétique pour leur génération le lien entre l'acte d'art et l'acte de foi ou de vie, cette fameuse théurgie dont Andrej Belyj et son frère en poésie, Aleksandr Blok, parlent dans leur correspondance. Entre le maître enfui et les prophètes de la « nouvelle conscience religieuse », il s'établit alors un véritable court-circuit, dont la marque est restée dans le recueil La religion de Tolstoj, paru en 1912, aux Éditions Put' (Le chemin). Andrej Belyj y déclare :

Dans son désir de dire l'indicible, Tolstoj est banni : de ce fait il est hors d'atteinte. Mais le sens authentique de cette exclusion de Tolstoj hors de l'aujourd'hui, le sens de son silence et de sa quête l'apparente aux bannis du monde entier ${ }^{1}$.

Et cette fuite de Tolstoj, qui l'apparentait aux grands ascètes et créateurs religieux de vie, avait toujours été le rêve des jeunes symbolistes, comme en témoigne l'ascendant qu'eut sur eux le poète Aleksandr Dobroljubov, le poète de la fuite dans le grand cosmos de la vie russe, celui qui avait fondé une communauté de frères, et dont les retours inopinés et brefs à la vie des villes, les visites impromptues à Brjusov ou à Belyj ont laissé un souvenir impérissable. Dans son Livre invisible (Iz knigi nevidimoj) Dobroljubov cite John Ruskin sur l'égalité des choses grandes et des choses petites aux yeux du Créateur, et l'égale part de mystère que grandes et petites choses comportent. Sur quoi Dobroljubov le prophète raconte ses errances de par la terre affamée, qui lui brûle la plante des pieds. Cet aspect de fuga mundi, de fuite hors du monde pécheur est aussi une composante, à l'évidence religieuse, de l'attente apocalyptique de la génération symboliste. Elle reprend d'ailleurs un thème qui avait également tourmenté Puškin, celui du poème de «l'Errant» («Strannik »), un poème de la dernière année de Puškin, et qui lui avait été inspiré par le poète puritain anglais John Bunyan. Le religieux rejoint ici l'art, l'anarchisme et l'ascétisme se conjuguent au prophétisme. L'art total des symbolistes est proche de la mort de l'art.

Autre tentation, et tentative, celle de Vjačeslav Ivanov et de son ami Georgij Čulkov : recréer un art total et religieux à la manière antique, un art mobilisant toute la cité comme le théâtre à Athènes ou les mystères à Delphes. Il s'ensuivit une violente querelle autour de « l'anarchisme mystique », une querelle qui confrontait nostalgiques de l'unité chrétienne du Moyen-Âge et nostalgiques de l'unité théâtrale de la cité antique. Ajoutons encore les «goethéens», ceux qui, sous 
l'influence du Goethe de la Seconde partie de Faust, et du Goethe théoricien des formes et des couleurs primitives sont en quête d'un substrat archaïque du monde naturel. Andrej Belyj passa par une phase aiguë de «goethéisme » conjugué à la théosophie du Dr Rudolf Steiner. Entre Emilij Medtner et lui éclata une très violente polémique sur l'exégèse de Goethe. Le résultat fut un combat quasiment médiéval où il s'agit pour Belyj de défendre l'organicisme mystique de Goethe contre les interprétations trop naturalistes de Medtner.

Derrière la violence de la polémique se cachait la quête d'un art plus que wagnérien, alliant le son, la couleur et la pensée - et ce fut l'apparition de ces deux prodiges : le Russe Skrjabin et le Lituanien Ciurlionis. Tous deux représentent un point extrême de rupture des frontières, d'invasion du total dans la conscience de l'homme. Mais ce «davantage d'art » va de pair avec un «plus d'art du tout », la vie vivante prenant le dessus, avec ses fortes pulsions de mort ! Et cela se manifestera dans l'attrait pour les aspects violents de la révolution russe, la répulsion envers les biens matériels et les biens spirituels, la quête d'une nudité première. L'oubli salvateur, ce Léthé où veut entrer Mihail Geršenzon, contrairement à son interlocuteur Vjačeslav Ivanov (dans leur Conversation des deux coins (Perepiska iz dvuh uglov) qui date de 1919), ou encore la joie mauvaise de Blok apprenant que Šahmatovo, la propriété familiale, a été pillée par les paysans. «Les Douze» («Dvenadcat'»), ce poème en bande dessinée où le Christ mène la troupe des pillards, c'est aussi, à sa façon, l'aboutissement de l' « Âge d'argent ». Et Belyj fait pendant avec son «Christ est ressuscité » («Hristos voskrese ») où tirent les mitrailleuses, précédant de peu ses Carnets d'un toqué (Zapiski čudaka) où il hurle à la mort, vociférant qu'il veut écrire comme un sabotier, c'est-à-dire saboter l'art ! Un rêve de destruction des limites, comme le rêve tolstoïen de gommer la frontière entre art et vie.

Bien entendu la part de l'occulte dans la culture du début du siècle est grande. D'ailleurs elle est de mieux en mieux étudiée, depuis l'ouvrage dirigé par Bernice Glatzer Rosental jusqu' au livre récent de Nikolaj Bogomolov². La gnose tourmente les esprits qui réclament hic et nunc le pourquoi du monde, et qui ne supportent pas de vivre avec des parts d'inconnu en attente. Cette impatience gnostique caractérise toute l'époque symboliste russe. Le poète Vjačeslav Ivanov, qui traduisit les «Hymnes à la nuit » de Novalis, écrit que Goethe avait dit du poète : « Empereur il n'était pas encore, mais allait devenir.» Comment comprendre : Napoléon allait devenir empereur, mais Novalis, en quel sens ? L'un et l'autre, explique Ivanov, veulent et réussissent à abattre les frontières, celles entre la révolution et la monarchie universelle en ce qui concerne Napoléon et, pour le poète mystique, celle entre l'individualisme et la «conciliarité » renouvelée du christianisme. Il s'agit de retrouver le grand chœur de la Foi globale et conciliaire de Dante. Renouveler l'exploit de Dante à l'heure où sont attelées ensemble Révolution et Hiérarchie, Liberté et Satiété ( $s$ voboda i sytost'́). 
Assurément Ivanov représente le versant conservateur de la démarche déstabilisatrice de l'Âge d'argent, conservateur en ce sens qu'il vise à une restauration totale de la Mémoire humaine : s'il veut et proclame la sortie extra muros de l'art, c'est pour retrouver les Idées Mères goethéennes, l'antique Mère, l'antique matrice de toutes choses. Il célèbre l'Extase, cela même qui est célébré dans le «Poème de l'Extase » de Skrjabin et cette «Ex-stase » doit nous ramener au bercail, c'est-àdire dans la «Maison du Père $»^{3}$. D'autres, et en particulier le grand destructeur philosophique de cet Âge, le philosophe Šestov, ne vont pas vers la Maison du Père, mais partent vers l'inconnu glacial.

Les deux attitudes ont néanmoins une commune racine: ne pas rester intra muros. Ni dans les murs de l'art, ni dans les murs de la vie. « Car tout le vivant naît de l'Extase et de la démence », écrit Vjačeslav Ivanov dans son article « Regard de Skrjabin sur l'art» («Vzgljad Skrjabina na iskusstvo») $)^{4}$. Comme Aleksandr Dobroljubov, le poète errant, Ivanov recourt à une distinction spinozienne : forma formata et forma formans, dans un article en italien. «Natura naturata, natura naturans », Dobroljubov intitule ainsi son second recueil. Il s'agit d'un concept de potentialité et d'accomplissement des potentialités, à la manière d'Aristote parlant des formes. Le mouvement ascendant et le mouvement descendant de l'acte créateur, que tous les symbolistes russes ont décrit, chacun à sa manière, Blok par exemple dans son poème «l'Aviateur» («Aviator»); et ils l'ont assimilé au mouvement de Zarathoustra, le prophète de vie de Nietzsche montant sur la montagne puis descendant dans la vallée. C'est au livre I de l'Éthique que l'on trouve, dans la troisième partie, le développement sur nature naturante et nature naturée, expressions venues de la scholastique ; Spinoza s'oppose avec force à la méthode de Descartes : l'intuition centrale de Spinoza est la quête de la béatitude plus que la quête de la vérité ou de la bonne méthode. Ce qui pour Descartes est substance, pour Spinoza est attribut : l'espace, l'étendue, le pensé sont des attributs, et tout conduit à la totalité. Il n'est de béatitude possible que celle qui se porte à l'unité de l'être, et c'est sur cette unité que la connaissance doit porter. On voit, en relisant Spinoza avec les principaux thèmes du symbolisme russe présents à l'esprit que l'âme humaine en tant que mode éternel du pensé qui est terminé par un autre mode éternel du pensé renvoie à l'infini, c'est-à-dire à l'entendement éternel et infini de Dieu. De même qu'il y a chez Spinoza substitution du point de vue de la production au point de vue de la création, il y a dans la quête infinie de tous les modes d'être du symbolisme russe une religiosité peu christique, fondée sur l'effort incessant des modes de l'être à persévérer dans leur être, et à produire de l'être. La synthèse des arts est une modalité de cette production indéfinie et infinie de toujours plus d'être dans le phénoménal. Et cette production repousse toujours davantage les limites de l'art, les amalgame, finalement les abolit.

3. L'expression est utilisée par Sergej Esenin dans son article sur Kotik Letaev. Elle désigne le retour en arrière, l'anabase vers les sources premières de l'Être.

4. Vjačeslav Ivanov, Sobranie sočinenij, t. III, Bruxelles, 1979, p. 175. 
L'influence de Mallarmé sur le symbolisme russe s'explique par cette recherche d'une religion sans acte de création initial. Une sorte d'après-midi solaire immobile où se conjuguent les lignes sonores et les lignes de couleur comme dans «L'aprèsmidi d'un faune ».

Et de faire aussi haut que l'amour se module

Évanouir du songe ordinaire de dos

Ou de flanc pur suivis avec mes regards clos, Une sonore, vaine et monotone ligne.

Bernard Weinberg dans son livre The limits of symbolism 5 interprète ce moment comme l'évanouissement des lignes de la passion et de la vision pour ne garder que la ligne sonore, de la musique. Brjusov et Annenskij ont traduit et étudié Mallarmé, deux poètes qui ne sont pas à nos yeux les plus représentatifs du symbolisme russe producteur de religieux. Et qui ont trahi Mallarmé, en le romantisant ${ }^{6}$. Bien plus que pour ces deux poètes, pour Alexandre Blok le principe de l'absence, principe mallarméen relié à celui du non finito ou encore du vague, est un principe poétique fondateur. La quête mallarméenne du livre unique transcendant les différents métiers et les différents arts a marqué ce symbolisme en déshérence qui cherche à capter les potentialités du phénomène dans son ensemble, dans sa totalité.

Voici Andrej Belyj à Munich où il a fui après l'épisode tragicomique, mais qui l'a conduit presque à la démence, de son amour pour Ljubov Blok, la «Belle Dame », la Vénus Astarté. L'orage est passé ; il reprend le texte de sa dernière Symphonie, la quatrième, symphonie en paroles et en silence qui se veut transcription du principe wagnérien musical des leitmotive, mais transcription d'un art dans l'autre. Il commente ainsi dans ses Mémoires, Entre deux révolutions (Meždu dvuh revoljucij) :

J'extirpai le texte déjà préparé jadis de ma Symphonie, pensant la retravailler, rêvant différents trucs techniques ; par exemple je désirais modeler le matériau de la phrase comme Wagner modèle le matériau de la mélodie ; je conçus une thématique selon une ligne rythmique sévère ; et des thèmes auxiliaires : deux femmes, l'une « ange », l'autre « démon », mais unies dans l'esprit du héros en une seule, non selon les règles de la logique, mais celles du contrepoint. Or la fable ne cédait pas à cette formule, la fable restait pour moi monolithique, tandis que la formule voulait la briser en deux mondes : le monde des hallucinations et le matériel. La confusion de ces deux mondes engendrait des illusions et dissociait le quotidien. La fable se recomposait dans le paradoxe du contrepoint. J'étais condamné à briser l'image dans un tourbillon de sonorités et d'éclats de lumière. Ainsi s'édifia «La coupe des tempêtes». Elle manifesta à jamais l'impossibilité de la «symphonie » en mots : à Munich, je crus pouvoir résoudre ce devant quoi avait reculé Mallarmé7.

5. Bernard Weinberg, The limits of symbolism ; studies of five modern French poets, Chicago, University of Chicago Press, 1966.

6. G. Nivat, « Spor o perevodnoj poezii », Etkindovskie čtenija, t. I, Saint-Pétersbourg, 2003, p. 1-15.

7. A. Belyj, Meždu dvuh revoljucij, Leningrad, 1934, p. 198. 
Plus tard Belyj tentera la fusion par un autre biais, présent déjà dans Petersburg, mais qui devient le principe créateur de Kotik Letaev : la cénesthésie, c'est-à-dire l'appréhension des différentes parties du corps depuis l'intérieur, une sorte de présence des parties dans le tout, qui amène à des états de déréalité. Kotik Letaev, un livre génial, ne fut pas compris par les lecteurs d'alors. La Coupe des tempêtes (Kubok metelej), plus axée sur la synesthésie ou traduction d'une série sensorielle dans une autre, reste, elle, un échec, mais un échec génial ${ }^{8}$.

Il y a un aveu d'impuissance presque congénital dans cette tentative symboliste de surmonter les limites, ou plutôt de remonter au moteur initial de toute forme, de toute nature, aux énergies, aux potentialités premières. Au cœur de cette symphonie de l'indéchiffrable se trouve la tempête, ou plutôt le tourbillon, la spirale en mouvement, la colonne de feu, c'est-à-dire la trace de Dieu lorsqu'il se montre au peuple juif, théophanie sous forme de spirale sonore, de tempête langagière.

O toi, tempête de neige ! boule blanche ; hurlement neigeux, rire écumeux, rumeur venteuse ! Tu es comme oiseau transparent, comme cygne qui s'enlève à l'empyrée!

$\mathrm{Tu}$ as rebondi par dessus les cloches qui se déversent sur nous. De ta plume lumineuse, de ta colonne neigeuse grommelle dans l'azur !

Et que hurle la cloche universelle ! appellant à la liturgie du monde : Prions pour la tempête !

Outre Vrubel' et Skrjabin qui sont les deux plus grands artistes de la synthèse des arts en ce début du siècle en Russie, il y a aussi le peintre compositeur lituanien Ciurlionis, né en 1875, mort en 1911. Auteur de vastes poèmes symphoniques où il ne fait usage que des cordes, renonçant tout à fait aux percussions, « La forêt » date de 1900, «La mer » date de 1907. « La forêt » fut donnée à Saint-Pétersbourg en 1912 pour le premier anniversaire de sa mort, car entre-temps les symbolistes russes avaient fait de lui un de leurs hérauts : ses toiles intitulées « Sonates », vastes mouvements colorés inspirés partiellement par le Japonais Hokusai, surimposées à ses symphonies mystérieuses permettaient de voir en lui un père de la synthèse des arts. Il fut proclamé Maître du symbolisme par Vjačeslav Ivanov dans une grande conférence sur «Ciurlionis et la synthèse des arts » publiée en 1911 dans la revue Apollon, puis reprise dans le recueil Sillons et bornes (Borozdy i meži) dont le titre même fait allusion au problème des «limites » du symbolisme ${ }^{10}$. Ivanov y salue l'art visionnaire de Ciurlionis, et y donne l'essentiel de sa théorie sur la synthèse des arts. Élaborer les éléments de la contemplation visuelle selon des lois venues de la musique, telle était la formule de Ciurlionis. Les énergies invisibles, très semblables aux énergies dont parlent les grands théologiens cappadociens, Grégoire de Nysse ou Nicolas Cabasilas, sont une extase, une sortie vers Dieu. Ivanov voit en Ciurlionis un visionnaire de l'âme du monde selon Goethe. L'extase, ou exode hors

8. A. Belyj, Kubok metelej, Četvertaja simfonija, Moscou, 1908.

9. Ibid., p. 41.

10. Vjačeslav Ivanov, Borozdy i meži, Moscou, 1916, p. 313 (reprint : Inkley, 1971). 
du phénomène (comme chez Skrjabin) s'effectue selon plusieurs chemins. Ivanov note pour finir que Ciurlionis, en tant que lituanien, est très proche des formes linguistiques les plus anciennes de l'indo-européen, donc plus apte que d'autres à remonter vers les Formes Mères. Cette ascension, ce sursum comme il dit, en reprenant un terme de la liturgie catholique (sursum corda) s'effectue selon une spirale ascendante.

À la spirale, précisément, Belyj attache une fonction philosophique toute particulière dans un article de la revue Logos. Ciurlionis sort du phénomène parce qu'il suit le chemin même de la religion, en quête de « réalisme cosmique ». Il recherche le grand lien perdu de la religion, et le recherche par la voie de la synthèse des arts et de la synesthésie. Voie qui est double, soit mythologique et intuitive, soit magique et cosmique. L'art, celui de Skrjabin ou de Ciurlionis, est un mystère syncrétique, rappelle Ivanov en reprenant une formule de Veselovskij, c'est-à-dire qu'il découle de l'unité de l'acte religieux et magique, et que la division des arts n'est venue qu'après. Dans une postface de 1914, Ivanov ajoute la note « aryenne » sur l'indo-européanisme de l'âme lituanienne de Ciurlionis et rajoute également une autre référence philosophique, empruntée cette fois à Leibniz, à sa Monadologie. Il s'agit de la notion d'entéléchie, qui est l'équivalent leibnizien des énergies cappadociennes, ou encore de la natura naturans, c'est-àdire la chaîne des finalités contenues dans le vivant, et qui conduisent de l'animal à l'esprit. «Tout est en harmonie et respire l'un dans l'autre. » Telle serait la formule philosophique du symbolisme russe dans sa recherche de l'unité première en amont du phénomène.

Ciurlionis en son temps a suscité en Russie un enthousisame énorme, et qui étonne un peu. Une récente exposition l'a remis pour un temps à la mode à Paris. Une partie de l'art symboliste a mal vieilli parce que ses ambitions philosophiques sont aujourd'hui mal comprises, surtout après la proclamation de la «mort de l'art». Les Symphonies d'Andrej Belyj sont peu lisibles quoique leur attrait magique subsiste. Vrubel' reste certes, tant par ses immenses tableaux et variations sur le Demon de Lermontov que par ses céramiques, ou ses fresques architecturales. Mais sa vision, qui a tant fasciné Blok, reste cependant marquée au sceau de l'époque, on y voit aujourd'hui un peu trop de « modern style ». Skrjabin est sûrement le plus grand des créateurs de la synthèse des arts. Mais il subsite aussi une tentative collective, qui dépasse chacun des acteurs du symbolisme russe : Ciurlionis, écrit Ivanov, était seul, incompris, et la solitude de ces créateurs, assez différente de la solitude des poètes maudits français est un thème qu'on peut dire complémentaire de cette quête mallarméenne de l'œuvre unique, steinerienne de ses formes premières. Nous voyons apparaître ici le thème de l'idiotie qui frappe le solitaire aventuré sur la voie de cette double quête. La folie de Nietzsche reste l'emblème de cette idiotie, de cette marque du destin. La folie de Vrubel', les crises de Belyj qui, dans les Notes d'un toqué, se présente lui-même comme un idiot et comme un savetier qui gâche l'objet d'art, qui « sabote » au sens littéral du terme. Ou encore, bien sûr, le naufrage de Blok à la fin de sa vie : Čukovskij fait allusion à la syphilis, maladie baudelairienne, tandis que le tabes dorsalis dévore le héros dans 
l'épilogue de Petersburg. Ne pourrait-on pas dire que cette hantise de l'idiotie est le pendant honteux de la quête de l'unité première ? A realibus ad realiora est la formule d'Ivanov, Blok la commente consciencieusement dans son Journal du 26 mars 1910 :

Le symbole doit devenir dynamique, se transformer en mythe. C'est la transition de la symbolisation à la symbolique. L'art théurgique est chose inévitable, et il ne se fait pas en son nom propre, mais au nom d'une chose sacrée : le monde en train de se construire. C'est l'heure de a realibus ad realiora. Un mythe est un jugement où le sujet est symbole et le prédicat est verbe (ou quelque chose d'intuitivement nouveau).

Et Blok d'ajouter que nous sommes tous les vibrations du Cavalier d'Airain, c'està-dire de l'Histoire, et d'une histoire dramatique :

Ce qui commence est une période de crise et de Jugement dernier. Ou bien le Verbe deviendra beau et sans âme (intérieurement anarchique), ou bien il deviendra pratique et vivant !11

Comme nous l'avons dit, Vrubel' indique par son destin tragique l'étape démoniaque du doute et de l'obscurcissement propre à toute mystique. Folie et idiotie ne sont pas loin : dans la poésie de Blok, c'est le panopticum, le musée de cire, la Baraque foraine, les derniers vers sur la «truie Russie ». Dans la phase d'obscurcissement, la phase démoniaque, la synesthésie joue aussi un grand rôle : violons, violet vroubelien, jappements discordants (vizg).

Très voisines de ces tentatives, s'effectuent au même moment celles de Schoenberg et de Kandinsky dans l'album du Blaue Reiter. Combien de tableaux de Kandinsky portent des noms musicaux : «Improvisation », « Composition », et ses pièces de théâtre: «Sonorité jaune», «Sonorité verte»! La peinture de Kandinsky introduit dans le tableau un élément venu de la musique : le temps, une sorte de perception de la temporalité à travers les étonnants signes que nous devons prendre le temps de déchiffrer sur l'espace du tableau. D'ailleurs Kandinsky eut des visions synesthéthiques, comme Belyj, il en rapporte une dans ses Regards sur le passé. On croirait lire quelque verset des Symphonies de Belyj :

Ce n'est que l'accord final de la symphonie qui porte chaque couleur au paroxysme de la vie et qui triomphe de Moscou tout entière en la faisant résonner comme le fortissimo final d'un orchestre géant. Le rose, le lilas, le jaune, le blanc, le bleu, le vert pistache, le rouge flamboyant des maisons, des églises, avec chacune sa mélodie propre ${ }^{12}$.

Lohengrin lui semble alors être la meilleure réalisation de cette Moscou-là.

11. Aleksandr Blok, Zapisnye knižki, Moscou, 1965, p. 169.

12. Wassily Kandinsky, Regards sur le passé et autres textes 1912-1922, Paris, Hermann, 1974. 
Je voyais mentalement toutes mes couleurs, elles étaient devant mes yeux. Des lignes sauvages, presque folles, se dessinaient devant moi. Je n'osais aller jusqu'à me dire que Wagner avait peint en musique «mon heure » de Moscou. Mais il m'apparut très clairement que l'art possédait une beaucoup plus grande force que je ne l'avais imaginé tout d'abord, et que de plus la peinture pouvait déployer les mêmes forces que la musique.

Kandinsky s'intéresse alors à cet exemple de Gesamstkunst qu'est la symphonie «Prométhée » de Skrjabin avec son clavier de couleurs. Un sommet de la tentative sera l'année 1912 et l'échange entre Schoenberg et Kandinsky. Schoenberg écrit alors à Kandinsky dans une lettre :

La Sonorité jaune me plaît énormément, c'est exactement ce que j'ai essayé de faire avec ma « Main heureuse ». [...] Seulement vous allez encore plus loin que moi dans le renoncement à toute pensée consciente, à toute action conventionnelle ${ }^{13}$.

Les deux œuvres des deux créateurs vont à l'extrême limite de l'art total, où un art en fait appel à un autre pour sortir de ses frontières, de son « sillon » et tenter une ascension plus énergique vers le Total. L'action scénique est faite de lumières qui changent, de mouvements, il n'y a aucune intrigue. Nous retrouvons ici ce « vague », ce « bon vague » dont nous parlions au début de ce propos. Les mystères ainsi créés invitent évidemment le spectateur-auditeur à devenir même une sorte de cocréateur de l'œuvre, tant le « bon vague » baudelairien invite à une collaboration. Ce que Vjačeslav Ivanov devait illustrer dans un propos de son échange avec Geršenzon dans la Correspondance d'un coin à l'autre :

Les hommes ne sauraient rien s'offrir de plus précieux que l'intime et persuasive révélation, ne serait-ce que de leurs pressentiments — ou des rudiments de leurs pressentiments - d'une conscience plus haute et plus spirituelle ${ }^{14}$.

Université de Genève

nivat.gm@wanadoo.fr

13. Arnold Schönberg, Wassily Kandinsky, Briefe, Bilder und Dokumente einer aussergewöhnlichen Begegnung, Munich, Deutscher Taschenbuch-Verlag, 1983 (je remercie Tamara Franzova de m'avoir indiqué cette publication).

14. V. Ivanov, M. Gerschenson, Correspondance d'un coin à l'autre. Préface par Olga Deschartes, Lausanne, L'Âge d'homme, 1979, p. 44. 